Notre Dame Journal of Formal Logic

Volume 34, Number 1, Winter 1993

\title{
The Amalgamation Property in Normal Open Induction
}

\author{
MARGARITA OTERO
}

\begin{abstract}
It is known that open induction (OI), the fragment of Peano arithmetic, fails to have the joint embedding property, a result due to Wilkie. On the other hand we have proved that if we require our models to be normal, that is, to be integrally closed in their fraction fields, the corresponding theory NOI extending OI, has the joint embedding property. Here we prove NOI does not have the amalgamation property.
\end{abstract}

1 Introduction Let $\mathcal{L}$ denote the first-order language of ordered rings based on the symbols $0,1,+,-, \cdot,<$. Open Induction (OI for short) is the fragment of Peano Arithmetic axiomatized by the axioms for discretely ordered rings together with the following axiom-scheme:

$$
\forall \bar{x}((\theta(\bar{x}, 0) \wedge \forall y \geq 0(\theta(\bar{x}, y) \rightarrow \theta(\bar{x}, y+1)) \rightarrow \forall y \geq 0 \theta(\bar{x}, y)),
$$

where $\theta(\bar{x}, y)$ is a quantifier-free $\mathscr{L}$-formula.

In this article rings are always commutative with 1.

A first point in the study of models of $\mathrm{OI}$ is the following algebraic characterization.

Theorem 1.1 (Shepherdson) Let $M$ be a discretely ordered ring. $M$ is a model of open induction if and only if for all $r$ in the real closure of the fraction field of $M$ there is an $a \in M$ such that $|r-a|<1$.

Shepherdson [6] proved several independence results for OI using this criterion. In particular, he showed that OI does not prove the normality axioms. We remind the reader that a domain is called normal if it is integrally closed in its fraction field.

Let NOI denote the extension of OI by adding the normality axioms, that is, for each $n \in \mathbf{N}^{*}$

$$
\forall \bar{z}, x, y\left(x, y \neq 0 \wedge x^{n}+z_{1} x^{n-1} y+\cdots+z_{n} y^{n}=0 \rightarrow \exists w(x=w y)\right) .
$$

Received July 23, 1992; revised December 14, 1992 
Associated with OI we have the following two theories.

DOR: axioms for discretely ordered rings.

(Ordered domains satisfying $\neg \forall x(0<x<1)$.)

ZR: $\quad$ DOR together with axioms for $\mathbf{Z}$-rings.

( $M$ is a $\mathbf{Z}$-ring if for every $n \in \mathrm{N}^{*}, M / n M \simeq \mathrm{Z} / n \mathbf{Z}$.)

We also have their normal counterparts NDOR and NZR, respectively.

Requiring normality for a discretely ordered ring, we get an arithmetic closer to that of $\mathbf{Z}$. We remind the reader that $\mathbf{Z}$ is normal and $\mathbf{Q}$ is algebraically closed in the fraction field of any normal discretely ordered ring. More remarkably van den Dries proved in [1] that Hilbert's Irreducibility Theorem holds for normal discretely ordered rings.

Using Shepherdson's criterion, it is easy to see that every (normal) model of $\mathrm{OI}$ is a (normal) Z-ring. Although the extension is strict, Wilkie proved in [7] that every model of ZR can be embedded in a model of OI. The key lemma in his construction is the following.

Lemma 1.2 (Wilkie) Let $M$ be a model of $Z R$, and $F$ its fraction field. Let $L$ be an ordered field dense in its real closure, extending $M$ and $|M|^{+}$-saturated. Let $r$ be an element of the real closure of $F$ such that $|r-a|>1$ for all $a \in M$. Then there is $a z$ in $L$ transcendental over $F$ such that $|r-z|<1$ and $M[z]$ is discretely ordered.

With the notation of the lemma we say that $M[z]$ has been obtained from $M$ by the Wilkie-construction (against $r$ ).

On the other hand, provided we have ring homomorphisms $\varphi_{p}: M \rightarrow \mathbf{Z}_{p}$ for each prime $p$ (where $\mathbf{Z}_{p}$ denotes the ring of $p$-adic integers) we define

$$
M_{\varphi}=\left\{\frac{a}{n}: a \in M, n \in \mathbf{N}^{*} \text { and } n \mid \varphi_{p}(a) \text { for each prime number } p\right\} .
$$

If $M$ is a model of DOR then $M_{\varphi}$ is a model of ZR (see [1]), and we say $M_{\varphi}$ has been obtained from $M$ by the $\hat{Z}$-construction.

Note that both the Wilkie- and the $\hat{\mathbf{Z}}$-constructions preserve normality. That is, with the above notation, if $M$ is normal then $M[z]$ and $M_{\varphi}$ are both normal (van den Dries [1]).

Now to prove Wilkie's embedding theorem we start with a (normal) model of ZR and iterate both the Wilkie- and $\hat{\mathbf{Z}}$-constructions to get a (normal) model of OI.

In [2], Macintyre and Marker do a systematic study of primes and their residue rings in (normal) models of OI. We shall use the following result later on.

Theorem 1.3 (Macintyre-Marker) Suppose $M$ is a (normal) discretely ordered ring equipped with homomorphisms $\varphi_{p}: M \rightarrow \mathbf{Z}_{p}$ for each prime number $p$, and $q$ a positive prime element of $M$ with $\varphi_{p}(q)$ a unit of $\mathbf{Z}_{p}$ for each prime number $p$. Then there is an embedding $M \rightarrow R$, where $R$ is a (normal) model of OI, such that the ideal $q R$ is maximal. Moreover any other prime $q^{\prime}$ of $M_{1}$ satisfying $\varphi_{p}\left(q^{\prime}\right)$ a unit of $\mathbf{Z}_{p}$ for each prime number $p$, keeps prime in $R$.

In Otero [5] we prove that models of NOI have the joint embedding property (JEP for short). Among the usually studied fragments of arithmetic this 
property is specific for NOI. For, as Wilkie showed, OI does not have JEP (see for example Otero [4]) and in [4] we proved that fragments of Peano Arithmetic where induction with (bounded) quantifiers is allowed do not have JEP either.

In [5] a natural question is left open: Does NOI have the amalgamation property? Here we give a negative answer to this question.

Theorem 1.4 The theory of normal open induction does not have the amalgamation property.

As is remarked in [5], to prove JEP for NOI (that is, amalgamation over $\mathbf{Z}$ ) we made use of the fact that any model of NOI is an end extension of $\mathbf{Z}$ and a flat Z-module. Here we get a counterexample to amalgamation producing two models $M$ and $M_{1}$ of NOI, with $M_{1}$ an extension of $M$ which is neither flat as $M$-module nor an end extension of $M$. Then for a suitable model $M_{2}$ of NOI extending also $M$ we show that $M_{1}$ and $M_{2}$ cannot be amalgamated over $M$ into a discretely ordered ring.

2 The construction We need the following two lemmas to prove the theorem.

Lemma 2.1 There is a normal model of open induction $M$ and $q_{1}, q_{2}$ distinct positive infinite primes in $M$ such that $\left(q_{1}, q_{2}\right)$ is a prime ideal of $M$ and $\left(q_{1}, q_{2}\right) \cap \mathbf{Z}=\{0\}$.

Lemma 2.2 Let $M$ be a discretely ordered ring and $F$ its fraction field. Let $L$ be an ordered field dense in its real closure, extending $M$ and $|M|^{+}$-saturated. Let $q_{1}$ and $q_{2}$ be two distinct positive infinite primes in $M$. Then

(i) If $\left(q_{1}, q_{2}\right) \cap \mathbf{Z}=\{0\}$ then there is an $x$ in $L$ transcendental over $F$ such that $M\left[x, q_{1} / x, q_{2} / x\right]$ is a discretely ordered ring.

(ii) If $\left(q_{1}, q_{2}\right)$ is a radical ideal of $M, M$ is normal and $x$ is transcendental over $F$, then $M\left[x, q_{1} / x, q_{2} / x\right]$ is also normal.

Proof of Theorem 1.4: Let $M$ be as in Lemma 2.1. By Lemma 2.2 there is a normal discretely ordered ring $R_{1}=M\left[x, q_{1} / x, q_{2} / x\right]$ extending $M$ with $x$ transcendental over the fraction field of $M$ (in particular $|x|>1$ ). Being a model of OI, $M$ is endowed with ring homomorphisms $\varphi_{p}: M \rightarrow \mathbf{Z}_{p}$ for each prime number $p$. We extend them to $R_{1}$ by $\varphi_{p}(x)=1$ for each such $p$. Now we iterate the $\hat{\mathbf{Z}}$ - and Wilkie-constructions to get a model $M_{1}$ of NOI extending $R_{1}$.

On the other hand $q_{1}$ and $q_{2}$ are distinct positive primes in $M$. So by Theorem 1.3 there is a normal model of open induction $M_{2}$ extending $M$ in which $q_{2}$ is maximal and $q_{1}$ is prime. Hence since they are distinct and positive, there exist $u$ and $v$ in $M_{2}$ such that $u q_{1}+v q_{2}=1$.

Let $M^{\prime}$ be any ordered ring amalgamating $M_{1}$ and $M_{2}$ over $M$. Then $1 / x=$ $u\left(q_{1} / x\right)+v\left(q_{2} / x\right)$ in $M^{\prime}$; therefore $M^{\prime}$ cannot have a discrete order.

Remark As we have mentioned above, $M_{1}$ is neither an end extension of $M$ nor a flat $M$-module. The first assertion is clear. Let us prove the second one. Suppose first $R_{1}$ is a flat $M$-module. Then

$$
\left(I_{1} \cap I_{2}\right) R_{1}=I_{1} R_{1} \cap I_{2} R_{1}
$$


for any pair of ideals $I_{1}, I_{2}$ of $M$ (see, for example, Theorem 2.7.4 in Matsumura [3]).

Let $I_{i}=\left(q_{i}\right)(i=1,2)$. Then $I_{1} \cap I_{2}=\left(q_{1} q_{2}\right)$ for $q_{1}$ and $q_{2}$ are prime in $M$. Now, $q_{1} q_{2} / x=q_{1} \cdot q_{2} / x=q_{2} \cdot q_{1} / x$ belongs to $q_{1} R_{1} \cap q_{2} R_{1}$, hence also belongs to $q_{1} q_{2} R_{1}$. Therefore

$$
q_{1} q_{2}=q_{1} q_{2} x \sum_{(i, j, k) \in J} a_{i j k} x^{k-(i+j)} q_{1}^{i} q_{2}^{j}=q_{1} q_{2} \sum_{k-(i+j)=-1} a_{i j k} q_{1}^{i} q_{2}^{j}
$$

for some $a_{i j k} \in M$ (where $J$ is a finite subset of $\mathbf{N}^{3}$ ). Therefore $1=a q_{1}+b q_{2}$ with $a, b \in M$. This is a contradiction, hence $R_{1}$ is not flat over $M$.

On the other hand $\left(q_{1}, q_{2}\right) \cap \mathbf{Z}=\{0\}$. Let $S$ denote $\mathbf{Z}^{*}$. Then

$$
M_{1} \subset S^{-1} R_{1}\left[z_{\nu} ; \nu \in \Delta\right]
$$

with the $z_{\nu}$ 's algebraically independent over the fraction field of $S^{-1} R_{1}$, so a similar argument shows that $M_{1}$ is not $M$-flat.

This last fact was the idea behind getting a counterexample for the amalgamation property for NOI; however, it is not used later on.

To prove Lemma 2.1 we need the following.

Lemma 2.3 Let $B$ be a model of $Z R$ and $q_{1}, q_{2}$ distinct positive infinite primes in $B$. Then

$$
\left(q_{1}, q_{2}\right) S^{-1} B \cap B=\left(q_{1}, q_{2}\right) B .
$$

Proof: For the nontrivial inclusion suppose $a \in B$ is such that $a n=u_{1} q_{1}+u_{2} q_{2}$ for some $n \in \mathbf{N}^{*}$ and $u_{1}, u_{2}$ in $B$. Since $q_{1}$ and $q_{2}$ are prime in $B$, their images $\varepsilon_{1, p}$ and $\varepsilon_{2, p}$ are units in $\mathbf{Z}_{p}$ for each $p$. Let $n=p_{1}^{m_{1}} \ldots \ldots p_{l}^{m_{l}}$ be the prime factorization of $n$ in $\mathbf{N}$. Get $s_{p} \in \mathbf{Z}, s^{p} \equiv\left(\varepsilon_{1, p}\right)^{-1}\left(\bmod p^{m}\right)$ in $\mathbf{Z}_{p}$ for each $p^{m} \in$ $\left\{p_{1}^{m_{1}}, \ldots, p_{l}^{m_{l}}\right\}$.

Let $\alpha_{i, p}=\varphi_{p}\left(u_{i}\right) \in \mathbf{Z}_{p}(i=1,2)$ and get $b \in \mathbf{Z}, b \equiv \alpha_{2, p} s_{p}\left(\bmod p^{m}\right)$ in $\mathbf{Z}_{p}$ for each $p^{m}$ as above.

Since $a=\left(u_{1} / n\right) q_{1}+\left(u_{2} / n\right) q_{2}=\left[\left(u_{1}+b q_{2}\right) / n\right] q_{1}+\left[\left(u_{2}-b q_{1}\right) / n\right] q_{2}$, it suffices to prove that $\alpha_{2, p}-b \varepsilon_{1, p} \equiv 0\left(\bmod p^{m}\right)$ and $\alpha_{1, p}+b \varepsilon_{2, p} \equiv 0\left(\bmod p^{m}\right)$ for each $p^{m}$ as above.

The first assertion is clear by the choice of $b$. For the second one note that $a \in B$ so $\alpha_{1, p} \varepsilon_{1, p}+\alpha_{2, p} \varepsilon_{2, p} \equiv 0\left(\bmod p^{m}\right)$, hence $\alpha_{1, p}+b \varepsilon_{2, p} \equiv \alpha_{1, p}+$ $\alpha_{2, p} s^{p} \varepsilon_{2, p} \equiv \alpha_{1, p}-s_{p} \alpha_{1, p} \varepsilon_{1, p} \equiv 0\left(\bmod p^{m}\right)$.

Proof of Lemma 2.1: Fix some ordered field $L$ dense in its real closure and $\aleph_{1^{-}}$ saturated. We shall construct such $M$ inside $L$. We begin with $A_{0}=\mathbf{Z}\left[q_{1}, q_{2}\right]_{\varphi}$ with $q_{1}, q_{2} \in L$ algebraically independent over $\mathbf{Q}, q_{1}>n$ for all $n \in \mathbf{N}$, and $q_{2}>\left|f\left(q_{1}\right)\right|$ for all $f(v) \in \mathbf{Z}[v]$. We define, for each prime number $p$, homomorphisms $\varphi_{p}: \mathbf{Z}\left[q_{1}, q_{2}\right] \rightarrow \mathbf{Z}_{p}$ by $\varphi_{p}\left(q_{i}\right)=\varepsilon_{i, p}$ for some fixed $\varepsilon_{i, p}$ unit of $\mathbf{Z}_{p}$ $(i=1,2)$. So $A_{0}$ is obtained from $\mathbf{Z}\left[q_{1}, q_{2}\right]$ and these $\varphi_{p}$ 's using the $\hat{\mathbf{Z}}$-construction.

Now, starting with this $A_{0}$ we get $M$ by iterating the Wilkie- and $\hat{\mathbf{Z}}$-construction; thus $A_{i+1}=A_{i}\left[z_{i}\right]_{\varphi}$ with $\varphi\left(z_{i}\right)=0$ and taking unions at limit stages. By stage $\omega \cdot \omega$ we have $M$, a model of OI which is normal since $\mathbf{Z}\left[q_{1}, q_{2}\right]$ is so and the Wilkie- and $\hat{\mathbf{Z}}$-constructions preserve normality. 
We prove by induction that $M$ has the required conditions. First, note that $\varphi_{p}\left(q_{1}\right)$ and $\varphi_{p}\left(q_{2}\right)$ are units of $\mathbf{Z}_{p}$ for each $p$, so $q_{1}$ and $q_{2}$ are also prime in $M$ (see Lemmas 3.3 and $3.11 \mathrm{~b}$ in [2]). Hence we have left to prove:

(a) $\left(q_{1}, q_{2}\right) A_{i} \cap \mathbf{Z}=\{0\}$ and

(b) $\left(q_{1}, q_{2}\right) A_{i}$ prime.

Let $S$ denote, as above $\mathbf{Z}^{*}$. $j=0$ :

(a) $\left(q_{1}, q_{2}\right) A_{0} \cap \mathbf{Z}^{*} \subset\left(q_{1}, q_{2}\right) \mathbf{Q}\left[q_{1}, q_{2}\right] \cap \mathbf{Z}^{*}=\varnothing$.

(b) $\left(q_{1}, q_{2}\right) S^{-1} A_{0}=\left(q_{1}, q_{2}\right) \mathbf{Q}\left[q_{1}, q_{2}\right]$ is a prime ideal, so if $u, v$ are in $A_{0}$ and $u v \in\left(q_{1}, q_{2}\right) A_{0}$, then $u \in\left(q_{1}, q_{2}\right) S^{-1} A_{0}$, say.

Therefore $u \in\left(q_{1}, q_{2}\right) S^{-1} A_{0} \cap A_{0}=\left(q_{1}, q_{2}\right) A_{0}$ by Lemma 2.3. $j=i+1: A_{i+1}=A_{i}\left[z_{i}\right]_{\varphi}$.

(a) Suppose $n \in\left(q_{1}, q_{2}\right) A_{i}\left[z_{i}\right]_{\varphi}$. Then $n=u\left(z_{i}\right) q_{1}+v\left(z_{i}\right) q_{2}$, so $n=$ $u(0) q_{1}+v(0) q_{2}$. Now applying Lemma 2.3 and induction we get $n=0$.

(b) By induction $\left(q_{1}, q_{2}\right)$ is a prime ideal of $A_{i}$, so $\left(q_{1}, q_{2}\right) A_{i}\left[z_{i}\right]$ is also prime in $A_{i}\left[z_{i}\right]$. On the other hand, since $\left(q_{1}, q_{2}\right) A_{i}\left[z_{i}\right] \cap \mathbf{Z}^{*}=\varnothing$ we have $\left(q_{1}, q_{2}\right) S^{-1} A_{i}\left[z_{i}\right]$ prime. By Lemma 2.3

$$
\left(q_{1}, q_{2}\right) A_{i}\left[z_{i}\right]_{\varphi}=\left(q_{1}, q_{2}\right) S^{-1} A_{i}\left[z_{i}\right] \cap A_{i}\left[z_{i}\right]_{\varphi},
$$

so $\left(q_{1}, q_{2}\right) A_{i+1}$ is a prime ideal.

Clearly (a) and (b) are preserved under taking unions.

Finally we prove Lemma 2.2. It is a generalization of Lemmas 3.22 and 3.23 in [2]. Their aim with those lemmas was to make reducible (in some extension) an irreducible element of $M$. In ours the aim is to have in some extension of $M$ a common factor of two primes of $M$.

Proof of Lemma 2.2: Get $M, q_{1}$ and $q_{2}$ as in Lemma 2.1 and $L \supset M$ as in the hypothesis of Lemma 2.2.

The proof of (i) is similar to that of Lemma 3.22 in [2]. We first get $x$ transcendental over $F$ satisfying the standard cut, that is, $x>m$ for all $m \in \mathbf{Z}$, and $x<a$ for all those $a \in M$ which satisfy $a>m$ for all $m \in \mathbf{N}$.

Suppose there is $f(u, v, w) \in M[u, v, w]$ such that $0<f\left(x, q_{1} / x, q_{2} / x\right)<1$. We write $f\left(x, q_{1} / x, q_{2} / x\right)=1 / x^{s} g(x)$ where $g(v) \in M[v]$ and $g(0) \neq 0$.

Let $f\left(x, q_{1} / x, q_{2} / x\right)=\sum_{l=-s}^{n} d_{l} x^{l}$ where $d_{l}=\sum_{k-(i+j)=l} a_{i j k} q_{1}^{i} q_{2}^{j}$, then $g(v)=\sum_{i=0}^{n+s} d_{i-s} x^{i}$.

Next we prove that there is an $l$ such that $\left|d_{l}\right|>m$ for all $m \in \mathbf{N}$. First, suppose there is $d_{l} \neq 0$ for some $l<0$. Then all the $(i, j)$ occurring in the summation of this $d_{l}$ must be either $i<0$ or $j<0$. This implies $d_{l} \in\left(q_{1}, q_{2}\right)$. Hence, either $d_{l}=0$ or $d_{l} \notin Z$. By discreteness of the order this last one implies $\left|d_{l}\right|>$ $m$ for all $m \in \mathbf{Z}$. Now suppose $d_{l}=0$ for all $l<0$, then $f\left(x, q_{1} / x, q_{2} / x\right) \in M[x]$ so one of the $d_{l}$ 's must not be in ZZ. Otherwise $f\left(x, q_{1} / x, q_{2} / x\right) \in \mathbf{Z}[x]$ which is a discretely ordered ring, contradicting $0<f\left(x, q_{1} / x, q_{2} / x\right)<1$.

The rest of the proof follows as in 3.22 of [2]. It is based on quantifier elimination in $K$ the real closure of $F$, and it also makes use of the prime factorization of $g(v)$ in $K(i)[v]$.

To prove (ii) we first make use of Lemma 3.23 in [2] to get $R=M\left[x, q_{1} / x\right]$ normal. To prove that $R_{1}=R\left[q_{2} / x\right]$ is also normal suffices to prove that $x R_{1}$ is a radical ideal of $R_{1}$ (see for example 3.17 in [2]). 
We have

$$
R_{1} / x R_{1} \simeq\left(M[x, u, v] /\left(u x-q_{1}, u x-q_{2}\right)\right) /(x) \simeq M[u, v] /\left(q_{1}, q_{2}\right) .
$$

Hence $R_{1} / x R_{1} \simeq\left(M /\left(q_{1}, q_{2}\right)\right)[u, v]$.

Now, since $\left(q_{1}, q_{2}\right)$ is a radical ideal of $M, M /\left(q_{1}, q_{2}\right)$ does not have nilpotent elements, therefore $\left(M /\left(q_{1}, q_{2}\right)\right)[u, v]$ does not have nilpotents either. This means $x R_{1}$ is radical.

Acknowledgment This note was written during a one year visit to the University of Illinois at Chicago. Research was supported by MEC/FPPI and CICYT/PB890379C0202 grants. I heartily thank David Marker for helpful suggestions on this paper and for his hospitality during my stay in Chicago.

\section{REFERENCES}

[1] van den Dries, L., "Some model theory and number theory for models of weak systems of arithmetic," pp. 346-362 in Model Theory of Algebra and Arithmetic, edited by L. Pacholski, J. Wierzejewski, and A. Wilkie, Springer-Verlag, Berlin, 1980.

[2] Macintyre, A. and D. Marker, "Primes and their residues: Rings in models of open induction," Annals of Pure and Applied Logic, vol. 43 (1989), pp. 57-77.

[3] Matsumura, H., Commutative Ring Theory, Cambridge University Press, Cambridge, 1989.

[4] Otero, M., "A note on the joint embedding property in fragments of arithmetic," Bulletin of the London Mathematical Society, vol. 110 (1992), pp. 417-423.

[5] Otero, M., "The joint embedding property in normal open induction," Annals of Pure and Applied Logic, forthcoming.

[6] Shepherdson, J., "A nonstandard model for a free variable fragment of number theory," Bulletin des l'Academie Polonaise des Sciences, vol. 12 (1964), pp. 79-86.

[7] Wilkie, A., "Some results and problems on weak systems of arithmetic," pp. 285296 in Logic Colloquium '77, North-Holland, Amsterdam, 1978.

Dept. Matemáticas

Universidad Autónoma de Madrid

Madrid, 28049

Spain 